\title{
Perfil ganador del premio extraordinario de la educación secundaria obligatoria en Galicia (España)
}

\author{
Winner profile of Extraordinary Prize of Secondary Education in Galicia (Spain)
}

\section{Perfil ganhador do Prêmio extraordinário de Ensino Secundário Obrigatório na Galícia (Espanha)}

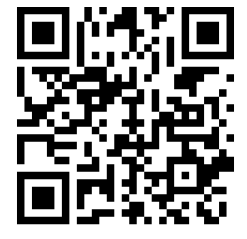

Recibido • Received • Recebido: 06 / 07 / 2017

Corregido • Revised • Revisado: 23 / 10 / 2018

Aceptado • Accepted • Aprovado: 06/ 02 / 2019

Resumen: Esta investigación tiene por objetivo analizar si existe un perfil determinado en las personas ganadoras del premio extraordinario de la educación secundaria obligatoria (ESO). Para ello, se compararán los resultados de la totalidad de las postulaciones a este premio con los ganadores y ganadoras en función del género, hábitat, titularidad y formación de sus padres y madres. La hipótesis de trabajo inicial es que se espera que no existan diferencias significativas ni en el colectivo que opta al premio, ni entre quienes lo ganan $-6,57 \%$ del alumnado presentado-; por ser el alumnado con mayor rendimiento académico. Para analizar estos perfiles, se han estudiado los rendimientos de todas las asignaturas del Premio Extraordinario de la ESO, de los 1.522 alumnos y alumnas que se presentaron para el período 2008-2013 en Galicia (España). La principal conclusión es que no se cumple la hipótesis de partida, dado que las variables analizadas sí influyen en el rendimiento del alumnado que se presenta al premio. El género lo hace en todas las asignaturas a favor del alumnado varón, el hábitat en el $57 \%$ de las materias, la titularidad en el $42,8 \%$ y la formación de los padres y madres en el $85,7 \%$. Teniendo en cuenta esto, el perfil sería el de chico de escuela privada y urbana, con padres y madres con formación superior.

Palabras claves: Premio extraordinario de la educación secundaria obligatoria ESO; perfil de 
doi: http://dx.doi.org/10.15359/ree.23-2.12

URL: http://www.una.ac.cr/educare

CORREO: educare@una.cr

alumnado; variables de desempeño académico; excelencia académica.

\begin{abstract}
This research aims to analyze if there is a profile of the students who win the "Extraordinary Prize of Secondary Education" (ESO) in Galicia (Spain). For this, the results of the totality of the students who competed for this prize will be compared according to their gender, habitat, ownership, and the level of formation of their parents. The initial working hypothesis was that if there would be no significant differences either in the group that opts for the prize, or between those who win it $-6.57 \%$ of the students competing-, because they are the students with the highest academic performance. In order to analyze these profiles, the study considered the performance in all the subjetcts of the Extraordinary ESO Prize of the 1,522 students who participated in the contest for the period 20082013 in Galicia (Spain). The main conclusion is that the initial hypothesis is not fulfilled, given that the variables analyzed do influence the performance of the students who compete for the award. Gender has influence on all subjects, favoring male students; habitat does on $57 \%$ of subjects, ownership on $42.8 \%$, and parent education on $85.7 \%$. Bearing this in mind, the profile would be that of a student from a private and urban school, with parents with higher education.
\end{abstract}

Keywords: Extraordinary Prize of Secondary Education; student profile; academic performance variables; academic excellence.

Resumo: Esta pesquisa tem como objetivo analisar a existência de um perfil determinado nas pessoas que ganham o prêmio extraordinário do ensino médio obrigatório (ESO). Para isso, se fará uma comparação dos resultados de todos os candidatos a este premio com os vencedores e vencedoras, de acordo com o gênero, residência, propriedade e nível de educação de seus pais e mães. A hipótese inicial do trabalho é que se espera que não haja diferenças significativas nem no grupo que escolhe o prêmio, nem entre aqueles que o recebem $-6,57 \%$ dos estudantes apresentados-, por serem estudantes com maior desempenho acadêmico. Para analisar esses perfis, estudamos o desempenho em todas as matérias do Prêmio Extraordinário do ESO, dos 1.522 alunos que foram apresentados para o período 2008-2013 na Galiza (Espanha). A principal conclusão é que a hipótese inicial não for cumprida, uma vez que se confirma que as variáveis analisadas influenciam no desempenho dos estudantes candidatos ao prêmio. O gênero, em todas as matérias favorece os estudantes do sexo masculino, a residência em $57 \%$ das matérias, a propriedade em $42,8 \%$ e a formação de pais e mães em $85,7 \%$. Tendo isso em conta, o perfil seria o de um menino de escola particular e urbana, com pais e mães com ensino superior.

Palavras chaves: Prémio extraordinário de ensino secundário obrigatório ESO; perfil do estudante; variáveis de desempenho acadêmico; excelência acadêmica.

\title{
Introducción
}

Los premios extraordinarios de enseñanza secundaria obligatoria (ESO) al rendimiento académico son convocados anualmente por la Consellería de Cultura, Educación y Ordenación Universitaria de la Xunta de Galicia con el objetivo de premiar y dar reconocimiento público a estudiantes de la ESO de la comunidad autónoma con los mejores resultados. El alumnado que se presenta a la convocatoria compite por hasta un máximo de 20 premios, debiendo satisfacer

Francisco Jesús Ferreiro-Seoane, Hugo Martínez-Ballesteros y Martha Camino-Santos\$s

Los artículos de la Revista Electrónica Educare del Centro de Investigación y Docencia en Educación de la Universidad Nacional, Costa Rica, se comparten bajo términos de la Licencia Creative Commons: Reconocimiento, No Comercial, Sin Obra Derivada 3.0 Costa Rica. Las autorizaciones adicionales a las aquí delimitadas se pueden obtener en el correo: educare@una.cr 
diversos requisitos entre los que destaca la obligatoriedad de tener una nota media de, al menos, 9.0 en su expediente académico (Xunta de Galicia. Consellería de Cultura, Educación e Ordenación Universitaria, 2017). Además de aportar su currículo, quienes aspiran a los premios extraordinarios de la ESO deben realizar una prueba estructurada en dos partes en la que tratarán cuestiones obligatorias de lengua gallega', castellana y primera lengua extranjera, ciencias sociales, geografía e historia, matemáticas y una cuestión optativa de entre las materias de biología-geología, física-química; educación plástica y visual, latín, música y tecnología. Deberán obtener una puntuación mínima de cinco puntos en cada materia, que se calificará entre 1 y 10 puntos con un decimal. A partir de los resultados obtenidos se calculará una nota final que permitirá conceder los premios en cuestión (Xunta de Galicia. Consellería de Cultura, Educación e Ordenación Universitaria, 2017).

Dado que el caso de estudio que se propone parte del análisis de un grupo de estudiantes que, por definición, se caracteriza por la excelencia académica, será conveniente explorar las variables que pueden influir en su desempeño.

Por lo expuesto, el objetivo principal de este artículo es comparar el perfil del alumnado ganador del premio extraordinario de la ESO en Galicia con el del resto de estudiantes presentados a la prueba, analizando si variables como el género, la titularidad del centro educativo, el hábitat, y la formación de sus padres, tienen influencia en los resultados. Como hipótesis inicial se espera que no existan diferencias de resultados en función de estas variables en el alumnado que se presenta al premio extraordinario de la ESO.

Sobre las variables que pueden incidir en el resultado académico del alumnado que se presenta al premio, la revisión de la bibliografía observa que existen investigaciones que aportan diversas justificaciones sobre las diferencias de rendimiento entre el estudiantado adolescente. Los que más popularidad han alcanzado en los últimos años son los informes PISA, estableciendo un ranking que clasifica a cada país según los conocimientos del alumnado. En estos trabajos, se destacan las variables socioeconómicas tratadas en el presente artículo. Mientras que algunos estudios confieren al autoconcepto y la motivación una mayor capacidad de predicción (Miñano y Castejón, 2008), otros, como los de Alonso-Tapia (2005); Barca, Peralbo y Brenlla (2004); Bermejo (2005); Cano (2000); Cuetos y Domínguez (2002), achacan una influencia importante en el rendimiento a las características socioeconómicas y familiares del alumnado, así como a la motivación hacia la escuela.

La incidencia del tipo de centro ha sido explorada por Mancebón-Torrubia, MartínezCaraballo y Pérez-Ximénez (2007), que consideraron variables socioeconómicas y antecedentes

${ }^{1}$ El estudiantado con exención en lengua gallega debidamente documentada estarán exentos de esta parte de la prueba (Xunta de Galicia. Consellería de Cultura, Educación e Ordenación Universitaria, 2017). 
doi: http://dx.doi.org/10.15359/ree.23-2.12

URL: http://www.una.ac.cr/educare

CORREO: educare@una.cr

personales del estudiantado de escuelas privadas-concertadas y públicas para concluir que, en términos de eficacia, los centros privados preparan mejor a sus estudiantes.

Los informes OCDE $(2009,2012)$ Ilaman la atención sobre la relevancia de la titularidad del centro educativo como factor clave en el análisis del rendimiento académico del estudiantado

Sobre el género, el informe realizado por la Swedish National Agency for Education (Skolverket, 2006) revela que los chicos obtienen notas más bajas que las chicas, confirma que las mujeres superan en todas las materias a los hombres. En Galicia, la Consellería de Cultura, Educación y Ordenación Universitaria señala que las alumnas muestran superioridad para las aptitudes verbales, mientras que los chicos destacan en matemáticas (Xunta de Galicia, 2012). Aunque los resultados expuestos por Skolverket (2006) y Xunta de Galicia (2012) han sido corroborados en los contextos educativos de otros países (Backhoff, Sánchez, Peón y Andrade, 2010; Gaviria, Martínez-Arias y Castro, 2004), otras experiencias de investigación sostienen que las diferencias en el rendimiento de estudiantes en virtud del género es insignificante (Acevedo y Rocha, 2011; Hyde, 2005; Matteucci y Mignani, 2011; Nowell y Hedges, 1998; Rodríguez-Cao, 2013; William, 2000).

Sobre el hábitat se ha argumentado que es un factor determinante en el rendimiento académico estudiantil (Kleinfeld, McDiarmid y Hagstrom, 1985). La lectura subyacente es que el hábitat, sea urbano o no urbano, influirá en los resultados esperados del alumnado de ESO.

\section{Método}

Para verificar si se cumple el objetivo, partiremos de los resultados obtenidos en todas las asignaturas, cinco obligatorias y la optativa, de todo el estudiantado que se presentó a la prueba del premio extraordinario de la ESO. Posteriormente, se identifica el grupo ganador. Estos dos grupos (ganador y la totalidad de postulante), se clasificarán según las variables: género, titularidad del centro, hábitat del alumnado y entorno educativo, usándose como principal técnica estadística el análisis de varianza unifactorial.

\section{Muestra}

La muestra total está formada por 1.522 estudiantes que optaron al premio en el período 2008-2013 en Galicia. Del total, 100 ganaron (6,57 \%), la nota media fue de 7,45 con una menor desviación típica $(0,5704)$. La nota media máxima fue de 9,45 y la nota mínima de 6,35. Lo anterior quiere decir que cualquier discente con resultados medios inferiores al notable ha ganado el premio, por estar dentro de los 20 mejores notas. La nota media del grupo no ganador ha sido de 5,16, con una desviación típica de 1,0402, una nota media máxima de 7,98 y notas mínimas 
de media de 1,72, lo que pone de manifiesto la dificultad de estos exámenes, ya que el que se presenta es un alumnado de sobresaliente en su etapa en la ESO.

\section{Instrumentos}

Para este trabajo han sido consideradas las calificaciones obtenidas por el estudiantado de ESO, clasificándolas en función de las variables de análisis: género, titularidad del centro, hábitat y formación de los sujetos progenitores. Los resultados han permitido comparar al grupo ganador del premio extraordinario de la ESO con el conjunto de estudiantes que compitieron por el premio. Los resultados analizados se corresponden con la nota media global de los exámenes de Lengua extranjera, Lengua castellana, Lengua gallega, Matemáticas, Ciencias sociales Geografía e Historia y, de entre las materias optativas, Biología-Geología y Física-Química.

\section{Procedimiento}

Metodológicamente, se aplica el análisis de varianza unifactorial, a través del estadístico $F$, y la prueba de Levene, que informa si el estadístico cumple o no el supuesto de varianzas iguales. En este trabajo, al haber sido identificados dos grupos: el total y el ganador, se aplican muestras independientes desglosando el género (chico-chica), el hábitat (rural-urbano), según la titularidad del centro (privada-pública) y en función de la formación de los padres y madres (con o sin estudios universitarios).

\section{Limitaciones metodológicas}

Como limitaciones metodológicas cabe señalar que se trata de un estudio circunscrito a los resultados obtenidos en Galicia, por lo tanto, es un trabajo desarrollado a nivel regional. Sin embargo, debe considerarse que, en España, las competencias educativas son transferidas por el gobierno central a las comunidades autónomas, siendo la Comunidad Autónoma de Galicia la que ostenta la potestad de convocar el premio, y establecer los criterios para su obtención. Esta reducción geográfica no le resta valor al trabajo, el cual es pionero en el estudio de este tipo de alumnado de excelencia académica a través de las pruebas objetivas del Premio Extraordinario de la ESO. Esta limitación podría servir, pues, como antesala para que estudios como este se extrapolen a otras comunidades autónomas españolas, abriendo la posibilidad de establecer marcos comparativos que permitan profundizar en el conocimiento de este tipo de alumnado.

Otro factor limitativo en este trabajo es el marco temporal. La investigación esta acotada en un período de cinco años (2008-2013). En el contexto español este período está irremediablemente ligado a una época de aguda crisis económica, por lo que sería interesante seguir estudiando al alumnado de excelencia, con el objeto de constatar si los resultados desgranados en esta investigación se verifican a lo largo del tiempo. 
doi: http://dx.doi.org/10.15359/ree.23-2.12

URL: http://www.una.ac.cr/educare

CORREO: educare@una.cr

Una tercera circunstancia que debe ser considerada es la selección de variables; se han escogido datos objetivos, no se ha nutrido la base de datos con variables subjetivas como podrían ser las de carácter psicológico, ambiental, la motivación o el profesorado.

\section{Resultados}

Como cabía esperar, el grupo ganador obtiene una media superior a la de quienes presentaron en todas las materias. En la Tabla 1, se puede comprobar cómo las diferencias son mayores en las asignaturas de ciencias con respecto a las que se producen en las lenguas,

Tabla 1: Calificaciones por materias del Premio Extraordinario ESO en Galicia (2008-2013)

\begin{tabular}{lcccccccc}
\hline \multicolumn{1}{c}{ Grupo } & No & $\begin{array}{c}\text { Media } \\
\text { total }\end{array}$ & $\begin{array}{c}\text { Lengua } \\
\text { extranjera }\end{array}$ & $\begin{array}{c}\text { Lengua } \\
\text { castellana }\end{array}$ & $\begin{array}{c}\text { Lengua } \\
\text { gallega }\end{array}$ & Matemática & CSGH & Optativa \\
\hline Que se presenta & 1.522 & 5,32 & 7,73 & 5,55 & 5,74 & 4,39 & 4,67 & 4,44 \\
Ganador & 100 & 7,45 & 8,31 & 7,41 & 7,41 & 7,59 & 6,88 & 7,31 \\
Diferencias G-P & 1.422 & 2,14 & 0,58 & 1,85 & 1,67 & 3,2 & 2,21 & 2,87 \\
\% Diferencias & $6,57 \%$ & $40,20 \%$ & $7,50 \%$ & $33,40 \%$ & $29,00 \%$ & $72,90 \%$ & $47,20 \%$ & $64,60 \%$ \\
\hline
\end{tabular}

Nota: Elaboración propia a partir de los datos facilitados por la Xunta de Galicia (2012).

siendo la materia de Matemáticas la que cuenta con la mayor diferencia, mientras que en Inglés la diferencia es mínima, tan solo medio punto y donde se obtiene la nota más alta. También debemos destacar que, en el grupo que se presentó, que ha obtenido una media de sobresaliente en toda la ESO, la media en Matemáticas, CSGH y la optativa (en la que predominan FísicaQuímica y Biología-Geología) es de suspenso.

\section{Género}

Las diferencias del rendimiento académico en función del género han sido objeto de numerosos estudios en las últimas décadas. Los últimos estudios revelan resultados más favorables en el caso del género femenino. Como fue avanzado, el informe de la agencia noruega de educación (Skolverket, 2006) verifica que las chicas obtienen mejores resultados que los hombres en todas las materias excepto en educación física. Otros (Backhoff et al., 2010; Gaviria et al., 2004) señalaron que las chicas son superiores en aptitudes verbales, mientras los chicos lo son en el ámbito matemático.

La Tabla 2 muestra que hay un mayor número de chicas que se presentan a la prueba 
(63\% del grupo presentado), reflejo de que obtienen mejores resultados durante la ESO. Por el contrario, son los chicos los que obtienen un mayor número de premios, suponiendo el $59 \%$ del total frente al $41 \%$ de las chicas. Analizando el porcentaje ganador frente al número del total presentado por género, se puede apreciar que el 10,5\% de los chicos presentados han resultado ganadores, frente a tan solo un $4,3 \%$ de las chicas. Por tanto, tanto en términos relativos como absolutos, los chicos han obtenido más premios que las chicas en el caso de la comunidad gallega y en el periodo de tiempo analizado, por lo que no se cumple la hipótesis inicial referida al género de que no debería haber diferencias en estudiantes más brillantes.

También, en la Tabla 2, se puede apreciar que, en el colectivo del total presentado, los estudiantes obtienen mejor rendimiento que ellas en todas las asignaturas. Resultados en consonancia con los expuestos en Ferreiro, Mato y Chao (2014). Al analizar el colectivo ganador, se producen diferencias a favor del género masculino en las asignaturas de ciencias y a favor del género femenino en las lenguas (Lengua extranjera, Lengua gallega y Lengua castellana).

Se vuelve a poner de manifiesto que la nota más alta se obtiene en la prueba de idioma extranjero, tanto para el género masculino como femenino. Se constata que, dentro del grupo ganador, el peor resultado se obtiene en Ciencias sociales, Geografía e Historia, tanto para las chicas como para los chicos. En cambio, en la muestra global, los chicos obtienen el peor resultado en las optativas, en las que predominan Física- Química, y Biología-Geología; mientras

Tabla 2: Calificaciones por materias en base al género del Premio Extraordinario de la ESO para el período 2008-2013 en Galicia

\begin{tabular}{lcccccccc}
\hline & No & $\begin{array}{c}\text { Media } \\
\text { total }\end{array}$ & $\begin{array}{c}\text { Lengua } \\
\text { extranjera }\end{array}$ & $\begin{array}{c}\text { Lengua } \\
\text { castellana }\end{array}$ & $\begin{array}{c}\text { Lengua } \\
\text { gallega }\end{array}$ & Matemáticas & CSGH & Optativa \\
\hline Grupo presentado & $\mathbf{1 . 5 2 2}$ & $\mathbf{5 , 3 2}$ & $\mathbf{7 , 7 3}$ & $\mathbf{5 , 5 5}$ & $\mathbf{5 , 7 4}$ & $\mathbf{4 , 3 9}$ & $\mathbf{4 , 6 7}$ & $\mathbf{4 , 4 4}$ \\
$\quad$ Hombre & 563 & 5,64 & 7,52 & 5,85 & 5,86 & 4,99 & 5,09 & 4,47 \\
$\quad$ Mujer & 959 & 5,14 & 7,37 & 5,57 & 5,65 & 3,56 & 4,51 & 4,11 \\
Diferencia M-H & 396 & $-0,5$ & $-0,15$ & $-0,27$ & $-0,21$ & $-1,43$ & $-0,58$ & $-0,35$ \\
\% diferencias & $\mathbf{7 3 , 8 \%}$ & $-8,90 \%$ & $-2,00 \%$ & $-4,60 \%$ & $-3,60 \%$ & $-28,70 \%$ & $-11,30 \%$ & $-7,90 \%$ \\
Grupo ganador & $\mathbf{1 0 0}$ & $\mathbf{7 , 4 5}$ & $\mathbf{8 , 3 1}$ & $\mathbf{7 , 4 1}$ & $\mathbf{7 , 4 1}$ & $\mathbf{7 , 5 9}$ & $\mathbf{6 , 8 8}$ & $\mathbf{7 , 3 1}$ \\
$\quad$ Hombre & 59 & $\mathbf{7 , 5 4}$ & 8,28 & 7,35 & 7,24 & 7,81 & 6,98 & 7,41 \\
$\quad$ Mujer & 41 & 7,34 & 8,34 & 7,43 & 7,46 & 7,11 & 6,66 & 7,01 \\
Diferencia M-H & -18 & $-0,2$ & 0,06 & 0,08 & 0,22 & $-0,7$ & $-0,32$ & $-0,4$ \\
\% diferencias & $-30,5 \%$ & $-2,70 \%$ & $0,80 \%$ & $1,10 \%$ & $3,10 \%$ & $-9,00 \%$ & $-4,50 \%$ & $-5,40 \%$ \\
\hline
\end{tabular}

Nota: Elaboración propia a partir de los datos facilitados por la Xunta de Galicia (2012). 
doi: http://dx.doi.org/10.15359/ree.23-2.12

URL: http://www.una.ac.cr/educare

CORREO: educare@una.cr

que las chicas alcanzan su peor resultado en Matemáticas.

Al aplicar el análisis de Varianza Unifactorial, cuyos resultados se pueden apreciar en el Apéndice $A$, se puede comprobar que, para el grupo presentado, el discente masculino obtiene mejor resultado en todas las materias de manera significativa, mientras que cuando se analiza el grupo ganador, solo se refleja en matemáticas. El resumen de datos se puede observar en la Tabla 2.

\section{Hábitat}

El hábitat del alumnado total que se presenta a los premios extraordinarios de la ESO es otra de las variables que, para muchos estudios, ejerce una fuerte influencia en el rendimiento académico (Kleinfeld et al., 1985).

En la Tabla 3, se puede observar cómo el número de estudiantes del ámbito urbanow $(1.028$ discentes procedentes de 20 municipios gallegos) es superior al del ámbito rural (494 discentes procedentes de 104 municipios gallegos), y de igual forma obtiene un mayor número ganador. En términos relativos, se aprecia que el alumnado del ámbito urbano cuenta con un porcentaje ganador sobre alumnado presentado superior $(7,4 \%)$, frente al $4,9 \%$ ganador proveniente del ámbito rural. Tampoco se cumple la hipótesis inicial de que no deberían existir diferencias entre los mejores promedios según procedan de la urbe o de poblaciones rurales. Tratando de dar una explicación a estos datos, hay que destacar que existe un mayor nivel de renta y formación de padres y madres de la urbe que del medio rural. De hecho, la mayor diferencia se produce en la asignatura Inglés. El nivel del alumnado en dicha lengua no solo depende del colegio, ya que en Galicia la mayoría de estudiantes va a académicas privadas para reforzar o mejorar sus competencias lingüísticas. Obtener clases privadas de inglés es más factible en zonas urbanas con padres y madres con mayor poder adquisitivo que en zonas rurales con menos academias de inglés y con sujetos progenitores con menor nivel de renta, los que no siempre pueden financiar clases extras ni estadías en países de habla inglesa.

Al desglosar los grupos entre quienes se presentaron y quienes ganaron, se observa cómo en el primero, el alumnado urbano supera en todas las asignaturas al del entorno rural, excepto en la nota del examen de gallego. En cambio, dentro del grupo ganador, se produce el efecto

\footnotetext{
${ }^{2}$ Se ha considerado como núcleo urbano aquellos municipios que superan los 20.000 habitantes o aquellos limítrofes con las grandes ciudades, ya que existen colegios que están ubicados en municipios pequeños al lado de grandes ciudades y, por tanto, el perfil de dichos colegios sería de chicos y chicas de ámbito urbano.
} 
Tabla 3: Calificaciones por materias en base al hábitat del Premio Extraordinario de la ESO para el período 2008-2013 en Galicia

\begin{tabular}{|c|c|c|c|c|c|c|c|c|}
\hline & No & $\begin{array}{l}\text { Media } \\
\text { total }\end{array}$ & $\begin{array}{l}\text { Lengua } \\
\text { extranjera }\end{array}$ & $\begin{array}{l}\text { Lengua } \\
\text { castellana }\end{array}$ & $\begin{array}{l}\text { Lengua } \\
\text { gallega }\end{array}$ & Matemáticas & $\mathrm{CSGH}$ & Optativa \\
\hline Grupo presentado & 1.522 & 5,32 & 7,73 & 5,55 & 5,74 & 4,39 & 4,67 & 4,44 \\
\hline Rural & 494 & 5,17 & 7,18 & 5,54 & 5,83 & 3,85 & 4,5 & 4,09 \\
\hline Urbano & 1.028 & 5,39 & 7,55 & 5,74 & 5,74 & 4,21 & 4,83 & 4,32 \\
\hline Diferencia R-U & 534 & $-0,22$ & $-0,37$ & $-0,2$ & 0,09 & $-0,36$ & $-0,33$ & $-0,23$ \\
\hline \% Diferencias & $108,09 \%$ & $-4,10 \%$ & $-4,90 \%$ & $-3,50 \%$ & $1,50 \%$ & $-8,60 \%$ & $-6,80 \%$ & $-5,30 \%$ \\
\hline Grupo ganador & 100 & 7,45 & 8,31 & 7,41 & 7,41 & 7,59 & 6,88 & 7,31 \\
\hline Rural & 24 & 7,6 & 8,37 & 7,65 & 7,12 & 7,54 & 7 & 7,53 \\
\hline Urbano & 76 & 7,42 & 8,29 & 7,3 & 7,4 & 7,52 & 6,8 & 7,16 \\
\hline Diferencia R-U & 52 & 0,18 & 0,08 & 0,36 & $-0,28$ & 0,02 & 0,2 & 0,36 \\
\hline$\%$ Diferencias & 216,66 & $2,40 \%$ & $1,00 \%$ & $4,90 \%$ & $-3,80 \%$ & $0,20 \%$ & $2,90 \%$ & $5,10 \%$ \\
\hline
\end{tabular}

Nota: Elaboración propia a partir de los datos facilitados por la Xunta de Galicia (2012).

contrario, es el alumnado del ámbito rural el que alcanza una media total superior, excepto en lengua gallega. Esto se puede ver analizado con más detalle en el desglose realizado en la Tabla 3.

De todas formas, lo relevante es la significatividad de dichas diferencias. En el Apéndice A se puede apreciar que, al analizar el resultado del alumnado presentado, se producen diferencias a favor del alumnado urbano en la nota global, Lengua extranjera, Lengua castellana, Matemáticas y CSHG; mientras que, al analizar al grupo ganador, las diferencias existentes no son significativas ni a favor del alumnado urbano ni del rural.

\section{Titularidad}

En relación con la variable titularidad del centro educativo, son interesantes las aportaciones (Bonal, 2002; Sánchez, 2008; Villarroya, 2001) que explican la diferencia en los resultados académicos alcanzados en los centros públicos respecto a los privados, en función de un menor acceso cuantitativo y cualitativo a recursos, y al hecho de que son estos centros los que acogen a estudiantes de familias de menor nivel socioeconómico y más estudiantes de origen inmigrante. En ese mismo sentido, otros trabajos apuntan al menor rendimiento de estudiantes inmigrantes (Calero y Waisgrais, 2009). Por otro lado, Perelman y Santin (2011) ponen en tela de juicio la inferioridad de los centros públicos, apoyándose en el "efecto composición" de estudiantes de 
doi: http://dx.doi.org/10.15359/ree.23-2.12

URL: http://www.una.ac.cr/educare

CORREO: educare@una.cr

dichos centros y de las escuelas privado-concertadas. Por lo expuesto, Calero y Escardíbul (2007) concluyen que no existe una diferencia significativa en relación con la titularidad del centro.

A continuación, a través de los datos analizados se observa en la Tabla 4 que el $61,8 \%$ de quienes se presentan al premio proceden de la escuela pública correspondientes a 194 instituciones académicas, frente al $38,2 \%$ de discentes de la escuela privada procedentes de 69 colegios de titularidad privada. El $60 \%$ ganador procede de la escuela pública, de tal manera que la relación porcentaje ganador por número de presentado es ligeramente superior a la escuela privada $6,9 \%$ frente al $6,4 \%$ de la escuela pública.

Al desglosar por asignaturas y entre el grupo presentado y ganador según titularidad del centro, se puede comprobar que, analizando toda la muestra, el rendimiento estudiantil de los colegios privados es ligeramente superior (Tabla 4), siendo significativa en Inglés y en

Tabla 4: Calificaciones por materias en base a la titularidad del centro en el Premio Extraordinario de la ESO para el período 2008-2013 en Galicia

\begin{tabular}{|c|c|c|c|c|c|c|c|c|}
\hline & No & $\begin{array}{c}\text { Media } \\
\text { total }\end{array}$ & $\begin{array}{l}\text { Lengua } \\
\text { extranjera }\end{array}$ & $\begin{array}{l}\text { Lengua } \\
\text { castellano }\end{array}$ & $\begin{array}{l}\text { Lengua } \\
\text { gallega }\end{array}$ & Matemáticas & $\mathrm{CSGH}$ & Optativa \\
\hline Grupo presentado & 1.522 & 5,32 & 7,73 & 5,55 & 5,74 & 4,39 & 4,67 & 4,44 \\
\hline Privada & 582 & 5,38 & 7,67 & 5,64 & 5,64 & 4,13 & 4,88 & 3,84 \\
\hline Pública & 940 & 5,29 & 7,28 & 5,7 & 5,79 & 4,07 & 4,63 & 3,46 \\
\hline Dif Pr-Pu & 358 & 0,09 & 0,39 & $-0,06$ & $-0,14$ & 0,07 & 0,25 & 0,38 \\
\hline \%Diferencias & $61,51 \%$ & $1,60 \%$ & $5,40 \%$ & $-1,00 \%$ & $-2,40 \%$ & $1,70 \%$ & $5,50 \%$ & $11,10 \%$ \\
\hline Grupo ganador & 100 & 7,45 & 8,31 & 7,41 & 7,41 & 7,59 & 6,88 & 7,31 \\
\hline Privada & 40 & 7,39 & 8,37 & 7,28 & 7,2 & 7,27 & 7,01 & 7,18 \\
\hline Pública & 60 & 7,51 & 8,27 & 7,45 & 7,42 & 7,7 & 6,75 & 7,3 \\
\hline Dif Pr-Pu & 20 & $-0,12$ & 0,1 & $-0,18$ & $-0,23$ & $-0,43$ & 0,26 & $-0,12$ \\
\hline \%Diferencias & $50 \%$ & $-1,60 \%$ & $1,30 \%$ & $-2,40 \%$ & $-3,00 \%$ & $-5,50 \%$ & $3,90 \%$ & $-1,60 \%$ \\
\hline
\end{tabular}

Nota: Elaboración propia a partir de los datos facilitados por la Xunta de Galicia (2012).

CSGH (Apéndice A), por tanto no se cumple la hipótesis inicial de que para niños y niñas tan sobresalientes no debería haber diferencias según el tipo de escuela donde hayan estudiado. La causa de este resultado, sobre todo el de inglés, radica en que los colegios privados, algunos de ellos internacionales, para diferenciarse de los públicos buscan otorgar un mayor nivel formativo y eso lo abanderan en el idioma inglés, con refuerzo de clases, con docentes que imparten parte 
de la docencia en el idioma extranjero o incluso con intercambios con colegios de otros países.

Al analizar el colectivo ganador, las diferencias entre estudiantes procedentes de la escuela pública y privada apenas son apreciables Tabla 4 y no tienen relevancia significativa en ninguna de las asignaturas (Apéndice $A$ ).

\section{Nivel formativo de los padres y madres}

El entorno familiar juega un importante papel en el rendimiento académico del alumnado, tal como han corroborado diversas investigaciones y publicaciones. La relación entre el nivel socioeconómicoy profesional delos progenitoresy progenitoras del estudiantadoyel rendimiento

Tabla 5: Número del grupo presentado y ganador con base en el entorno familiar del Premio Extraordinario de la ESO para el período 2008-2013 en Galicia

\begin{tabular}{lcccccc}
\hline & Sin estudios & ESO & FP & Bachillerato & Universitarios & Total \\
\hline N.o grupo presentado & 20 & 118 & 186 & 123 & 1.075 & 1.522 \\
N.o grupo ganador & & 5 & 6 & 4 & 85 & 100 \\
\% presentado/total & $1,30 \%$ & $7,80 \%$ & $12,20 \%$ & $8,10 \%$ & $70,60 \%$ & $100,00 \%$ \\
\% ganador/ total & $0,00 \%$ & $5,00 \%$ & $6,00 \%$ & $4,00 \%$ & $85,00 \%$ & $100,00 \%$ \\
\% ganador/ presentado & & $4,20 \%$ & $3,20 \%$ & $3,30 \%$ & $7,90 \%$ & $6,60 \%$ \\
Nota media presentado & 5,04 & 5,09 & 5,26 & 4,85 & 5,41 & 5,32 \\
Nota media ganador & & 7,85 & 7,46 & 7,11 & 7,59 & 7,45 \\
Di nota ganador-total & & 2,76 & 2,2 & 2,26 & 2,19 & 2,14 \\
\hline
\end{tabular}

Nota: Elaboración propia a partir de los datos facilitados por la Xunta de Galicia (2012).

académico ha sido explorado por Casullo (2002) y Encinas, Díaz, Noriega y Estrada (2009) quienes sostienen que mejores resultados académicos estarán ligados a la pertenencia a familias de mayor poder adquisitivo. Como se puede observar en la Tabla 5, el entorno familiar predominante entre el alumnado que se presentó al premio es el de madres o padres universitarios, en más del 70,6 $\%$ del total de estudiantes. Esta cifra es todavía mayor si nos centramos en el grupo ganador, pues el $85 \%$ proviene de un entorno familiar caracterizado por padres o madres con estudios superiores. Analizando la nota media de quienes se presentaron a la prueba, esta es superior en 
doi: http://dx.doi.org/10.15359/ree.23-2.12

URL: http://www.una.ac.cr/educare

CORREO: educare@una.cr

aquellos grupos que tienen familias con formación universitaria; sin embargo, en el caso ganador, el resultado muestra sus madres o padres tienen estudios secundarios $(7,85)$ han obtenido una nota media superior que quienes tienen padres o madres con estudios universitarios; si bien se debe matizar este dato, ya que solo han sido cinco personas ganadoras cuyos padres o madres tienen formación en la ESO, frente a los 85 con formación universitaria $(7,59)$.

Tabla 6: Resumen del rendimiento del premio extraordinario de la ESO por distintas variables y materia para el período 2008-2013

\begin{tabular}{|c|c|c|c|}
\hline Rendimiento académico & $\begin{array}{c}\text { Materias entre el } \\
\text { alumnado presentado }\end{array}$ & $\begin{array}{l}\text { Materias entre alumnado } \\
\text { ganador del premio }\end{array}$ & Véase \\
\hline \multirow{7}{*}{$\begin{array}{l}\text { El alumnado de género masculino tiene una } \\
\text { media superior "significativa" en los siguientes } \\
\text { exámenes }\end{array}$} & 1. Nota global media & Matemáticas & \multirow{7}{*}{ Apéndice $A$} \\
\hline & 2. Lengua extranjera & & \\
\hline & 3. Lengua castellana & & \\
\hline & 4. Lengua gallega & & \\
\hline & 5. Matemáticas & & \\
\hline & 6. CSGH & & \\
\hline & 7. Optativas & & \\
\hline \multirow{4}{*}{$\begin{array}{l}\text { El alumnado de hábitat urbano tiene una } \\
\text { media superior "significativa" en los siguientes } \\
\text { exámenes }\end{array}$} & 1. Nota global media & & \multirow{4}{*}{ Apéndice $A$} \\
\hline & 2. Lengua extranjera & & \\
\hline & 3. Matemáticas & & \\
\hline & 4. CSGH & & \\
\hline \multirow{3}{*}{$\begin{array}{l}\text { El alumnado de un centro privado tiene una } \\
\text { media superior "significativa" en los siguientes } \\
\text { exámenes }\end{array}$} & 1. Lengua extranjera & & \multirow{3}{*}{ Apéndice $A$} \\
\hline & 2. $\mathrm{CSGH}$ & & \\
\hline & 3. Optativa & & \\
\hline \multirow{6}{*}{$\begin{array}{l}\text { El alumnado con padres o madres con } \\
\text { formación universitaria tiene una media } \\
\text { superior "significativa" en los siguientes } \\
\text { exámenes }\end{array}$} & 1. Nota global media & Lengua extranjera & \multirow{6}{*}{ Apéndice $A$} \\
\hline & 2. Lengua extranjera & & \\
\hline & 3. Lengua castellana & & \\
\hline & 4. Lengua gallega & & \\
\hline & 5. Matemáticas & & \\
\hline & 6. $\mathrm{CSGH}$ & & \\
\hline
\end{tabular}

Nota: Elaboración propia a partir de los datos facilitados por la Xunta de Galicia (2012). 
Dado que discentes sin madres o padres universitarios representan el $29,4 \%$, se han agrupado en un colectivo denominado "padres o madres sin formación universitaria", para compararlo a través del análisis unifactorial con progenitores y progenitoras con formación superior, tanto para quienes se presentan como para el grupo ganador. El resultado es que el alumnado con padres o madres que tienen formación superior obtiene un rendimiento significativamente superior en todas las asignaturas menos la optativa, mientras que, si nos centramos en el colectivo ganador, esto se manifiesta solo en la nota de lengua extranjera (Apéndice A).

A modo de resumen, en la Tabla 6 se muestra, para cada una de las variables analizadas (género, hábitat, titularidad y entorno familiar), en qué materias obtiene una media superior cada uno de los grupos analizados, tanto del total de estudiantes que se presentó como del grupo ganador. En la última columna de la Tabla 6 se especifica en qué apéndice se muestra la información estadística desglosada.

\section{Conclusión}

Solo el 6,57\% del total presentado al premio extraordinario de la ESO obtiene el premio, lo que pone de manifiesto la alta dificultad de obtenerlo, dado que para presentarse hay que tener una media mínima de sobresaliente en toda la ESO.

La nota media obtenida por quienes se presentan al premio es de 5,32, sorprendiendo que exista una nota media de suspenso en Matemáticas $(4,39), \operatorname{CSGH}(4,67)$ y en la optativa $(4,44)$, en la que predomina Física-Química y Biología-Geología. La causa de que el alumnado con media de sobresaliente suspenda los exámenes es el alto nivel de exigencia de esas pruebas. Además, también influye que las pruebas tengan lugar en octubre del año siguiente a la finalización del curso, y hay discentes que no las preparan específicamente. Se aprueba en las lenguas, destaca el resultado en Inglés $(7,73)$, aspecto que va en consonancia con la preocupación de la sociedad gallega de dominar dicho idioma. En el colectivo ganador, las diferencias entre asignaturas son menores, donde la mayoría es la nota media de notable.

Aunque no cabría esperar diferencias significativas en función del género, hábitat, titularidad o formación académica de los padres o madres, sí se aprecian algunas.

En lo que al género se refiere, se ha observado que los chicos obtienen de manera significativa un mayor rendimiento en todas las asignaturas, y dentro del colectivo ganador, en matemáticas, para este período. Por tanto, en general, las chicas destacan claramente en la ESO, pero cuando nos centramos en el colectivo más sobresaliente, los chicos pueden superar a las chicas como ocurre en esta ocasión.

En cuanto al hábitat, el alumnado del mundo urbano, que es mayoritario en la prueba, supera de manera significativa al que procede del ámbito rural en la nota global, en Lengua 
doi: http://dx.doi.org/10.15359/ree.23-2.12

URL: http://www.una.ac.cr/educare

CORREO: educare@una.cr

extranjera, Matemáticas y CSGH. Sin embargo, no se aprecian diferencias significativas en el colectivo ganador del premio. Relacionándolo con otras variables como el nivel formativo, renta de la familia y medios como escuelas de idiomas, academias privadas de inglés, estadías en el extranjero, se dan mayores posibilidades en las ciudades que en el mundo rural.

Respecto a la variable titularidad, en solo tres asignaturas (Lengua extranjera, CSGH y optativa) se producen diferencias significativas a favor del alumnado del colegio privado y ninguna en el grupo ganador. La explicación en lengua extranjera es razonable al considerar que los colegios internacionales suelen ser privados.

Al analizar la influencia del nivel formativo de los padres o madres, se aprecia que se refleja de manera relevante en todas las asignaturas, excepto en las optativas, una inclinación a favor del alumnado con familias con formación universitaria, reduciéndose dicha significatividad a Lengua extranjera, al referirnos al grupo ganador del premio.

Para finalizar, enlazando el objetivo del trabajo de analizar si existe un perfil determinado en las personas que se presentan y ganadoras del premio extraordinario de la ESO, podemos afirmar que no se cumple la hipótesis de partida, dado que existen diferencias notables en el rendimiento académico del grupo presentado, en relación con variables como género, hábitat, titularidad y entorno familiar. El perfil resultante sería el de jóvenes de hábitat urbano, que proviene de un colegio privado y cuyos padres o madres poseen estudios universitarios. Si nos centramos en el colectivo ganador, no existe un perfil de estudiantado, a pesar de que el chico supere a la chica en Matemáticas, y quienes tienen madres o padres universitarios superen en inglés a quienes no les tienen.

Como reflexión final, el equipo autor de este texto pensamos que estos premios ayudan a mejorar el nivel académico de cada discente y nos parece acertado que se convoquen estos premios. De hecho, los premios extraordinarios de la educación secundaria obligatoria suponen un mérito oficial y académico a las capacidades y esfuerzo del alumnado, además de otorgarles un premio económico de 750 euros. Ahora bien, es una prueba voluntaria que hace el gobierno regional de Galicia y que complementa las pruebas obligatorias que han de realizar los grupos discentes para obtener la titulación de la ESO. Dada su notoriedad e importancia para el currículo de estudiantes recomendaríamos que se convocaran en todas las regiones de España y a nivel nacional.

\section{Referencias}

Alonso-Tapia, J. (2005). Motivaciones, expectativas y valores-intereses relacionados con el aprendizaje: El cuestionario MEVA. Psicothema, 17(3), 404-411. Recuperado de http:// www.psicothema.com/psicothema.asp?id=3120

Acevedo, C. G. y Rocha, F. (2011). Estilos de aprendizaje, género y rendimiento académico. Revista Estilos de Aprendizaje, 8(8), 1-16. Recuperado de https://www2.uned.es/ 
revistaestilosdeaprendizaje/numero 8/articulos//sr 8 articulo 5.pdf

Backhoff,E.,Sánchez, A.,Peón, M.y Andrade, E. (2010).Comprensión lectoray habilidades matemáticas de estudiantes de educación básica en México: 2000-2005. Revista Electrónica de Investigación Educativa, 12(1), 2-18. Recuperado de https://redie.uabc.mx/redie/article/view/242/402

Barca, A., Peralbo, M. y Brenlla, J. C. (2004). Atribuciones causales y enfoques de aprendizaje: La Escala Siacepa. Psicothema, 16(1), 94-103. Recuperado de http://www.psicothema.com/ pdf/1167.pdf

Bermejo, V. (2005). Microgénesis y cambio cognitivo: Adquisición del cardinal numérico. Psicothema, 17(4), 559-562. Recuperado de http://www.psicothema.com/pdf/3145.pdf

Bonal, X. (2002). El balance público-privado en el sistema de enseñanza español: Evolución y efectos sobre las desigualdades educativas. Educar, 29, 11-29. Recuperado de http:// educar.uab.cat/article/view/v29-bonal/297

Calero, J. y Escardíbul, J.-O. (2007). Evaluación de servicios educativos: El rendimiento en los centros públicos y privados medido en PISA-2003. Hacienda Pública Española, 183(4), 33-66.

Calero, J.yWaisgrais, S. (2009). Factores de desigualdad en la educación española:Una aproximación a través de las evaluaciones de PISA. Papeles de economía española, 119, 86-98.

Cano, F. (2000). Diferencias de género en estrategias y estilos de aprendizaje. Psicothema, 12(3), 360-367. Recuperado de http://www.psicothema.es/pdf/343.pdf

Casullo, M. M. (Compiladora). (2002). Evaluación del bienestar psicológico en Iberoamérica. Buenos Aires: Paidós.

Cuetos, F. y Domínguez, A. (2002). Efecto de la pseudohomofonía sobre el reconocimiento de palabras en una lengua de ortografía transparente. Psicothema, 14(4), 754-759. Recuperado de http://www.psicothema.com/pdf/794.pdf

Encinas, D. M., Díaz, C. I, Noriega, J. Á. y Estrada, D. Y. (2009). Locus de control y logro académico en dos tipos de ambiente de enseñanza para estudiantes universitarios. Pesquisas e Práticas Psicossociais, 3(2), 167-174. Recuperado de http://www.ufsj.edu.br/portal-repositorio/ File/Encinas etal.pdf

Ferreiro, F. J., Mato, M. D. y Chao, R. (2014). Análisis del rendimiento académico por género medido a través del premio extraordinario de la ESO. Revista de Investigaciones Políticas y Sociales (RIPS), 13(2), 53-76. Recuperado de http://www.usc.es/revistas/index.php/rips/ article/view/2086/2449

Gaviria, J.L., Martínez-Arias, R.y Castro, M. (2004). Un estudio multinivel sobre los factores de eficacia escolar en países en desarrollo: El caso de los recursos en Brasil. Education Policy Analysis 
doi: http://dx.doi.org/10.15359/ree.23-2.12

URL: http://www.una.ac.cr/educare

CORREO: educare@una.cr

Archives, 12(20), 1-31. Recuperado de https://epaa.asu.edu/ojs/article/viewFile/175/301

Hyde, J. (2005). The gender similarities hypothesis. American Psychologist, 60(6), 581-592. doi: https://doi.org/10.1037/0003-066X.60.6.581

Kleinfeld, J. S., McDiarmid, G. W. y Hagstrom, D. (1985). Alaska's small rural high schools: Are they working? (Report Series) ISER, 22(3), 1-16. Recuperado de http://www.iser.uaa.alaska.edu/ Publications/formal/arsecs/ARSEC XXII 3 AKRural High Schools.pdf

Mancebón-Torrubia, M. J., Martínez-Caraballo, N. y Pérez-Ximénez, D. (Julio, 2007). Un análisis de la calidad percibida por los estudiantes en los centros públicos y privados de enseñanza secundaria. En XVI Jornadas de la Asociación de Economía de la Educación. Gran Canaria, España. Recuperado de http://works.bepress.com/domingoperez/4/

Matteucci, M. y Mignani, S. (2011). Gender differences in performance in mathematics at the end of lower secondary school in Italy. Learning and Individual Differences, 21(5), 543-548. doi: https://doi.org/10.1016/j.lindif.2011.03.001

Miñano, P.y Castejón, J. L. (2008). Capacidad predictiva de las variables cognitivo-motivacionales sobre el rendimiento académico. Revista Electrónica de Motivación y Emoción, 11(28), 1-13. Recuperado de http://reme.uji.es/articulos/numero28/article4/article4.pdf

Nowell, A. y Hedges, L. V. (1998). Trends in gender differences in academic achievement from 1960 to 1994. Sex Roles, 39(1-2), 21-43. doi: https://doi.org/10.1023/A:1018873615316

OCDE. (2009). OCDE (2010) Pisa 2009 results: Overcoming social background. Equity in learning opportunities and outcomes (Vol. II), París: Autor. Recuperado de https://www.oecd.org/ pisa/pisaproducts/48852584.pdf

OCDE. (2012) PISA 2012. Programa para la evaluación internacional de los alumnos. Informe español (Vol. I: Resultados y contexto). Madrid: MEC. Recuperado de https:// www.mecd.gob.es/dctm/inee/internacional/pisa2012/pisa2012lineavolumeni. pdf?documentld=0901e72b81786310

Perelman, S.y Santin, D. (2011). Measuring educational efficiency at student level with parametric stochastic distance functions: An application to Spanish PISA results. Education Economics, 19(1), 29-49. doi: https://doi.org/10.1080/09645290802470475

Rodríguez-Cao, L. (2013). Muller e superdotación. En M. D. Mato, R. Chao y R. Suárez (Coords.), As mulleres nas artes e nas ciencias. Reflexións e testemuñas (Vol. I, pp. 191-197). A Coruña: Publicacións de centros e departamentos da UDC.

Sánchez, A. P. (2008). Efectos de la inmigración en el sistema educativo: El caso español (Tesis doctoral). Universidad de Barcelona, Barcelona. 
Apéndice A: Estadísticas de grupo y prueba de muestras independientes de las notas obtenidos para el período 2008-2013 en el premio extraordinario de la ESO

\begin{tabular}{|c|c|c|c|c|c|c|c|}
\hline \multicolumn{8}{|c|}{ Según género } \\
\hline \multicolumn{2}{|c|}{ Grupo que se presenta } & $\mathrm{N}$ & Media & F. & Sig. & Prueba de Levene & Sig. (bilateral) \\
\hline \multirow[t]{2}{*}{ Media global } & $\mathrm{H}$ & 563 & 5,638 & & & & \\
\hline & M & 959 & 5,139 & 3,364 & 0,067 & Se asumen varianzas iguales & 0 \\
\hline \multirow[t]{2}{*}{ L. Extranjera } & $\mathrm{H}$ & 563 & 7,521 & & & & \\
\hline & $\mathrm{M}$ & 959 & 7,374 & 4,571 & 0,033 & No se asumen varianzas iguales & 0,04 \\
\hline \multirow[t]{2}{*}{ L. Castellana } & $\mathrm{H}$ & 563 & 5,845 & & & & \\
\hline & M & 959 & 5,574 & 0,865 & 0,352 & Se asumen varianzas iguales & 0,007 \\
\hline \multirow[t]{2}{*}{ L. Gallega } & $\mathrm{H}$ & 563 & 5,864 & & & & \\
\hline & M & 959 & 5,653 & 0,449 & 0,503 & Se asumen varianzas iguales & 0,028 \\
\hline \multirow[t]{2}{*}{ Matemáticas } & $\mathrm{H}$ & 563 & 4,994 & & & & \\
\hline & M & 959 & 3,562 & 3,109 & 0,078 & Se asumen varianzas iguales & 0 \\
\hline \multirow[t]{2}{*}{$\mathrm{CSGH}$} & $\mathrm{H}$ & 563 & 5,087 & & & & \\
\hline & $M$ & 959 & 4,511 & 7,324 & 0,007 & No se asumen varianzas iguales & 0 \\
\hline \multirow[t]{2}{*}{ Optativa } & $\mathrm{H}$ & 563 & 4,465 & & & & \\
\hline & $\mathrm{M}$ & 959 & 4,112 & 5,081 & 0,024 & No se asumen varianzas iguales & 0,005 \\
\hline \multicolumn{2}{|l|}{ Grupo ganador } & $\mathrm{N}$ & Media & F. & Sig. & Prueba de Levene & Sig. (bilateral) \\
\hline \multirow[t]{2}{*}{ Media global } & $\mathrm{H}$ & 59 & 7,541 & & & & \\
\hline & $M$ & 41 & 7,341 & 0,391 & 0,533 & Se asumen varianzas iguales & 0,088 \\
\hline \multirow[t]{2}{*}{ L. Extranjera } & $\mathrm{H}$ & 59 & 8,281 & & & & \\
\hline & $M$ & 41 & 8,344 & 1,022 & 0,315 & Se asumen varianzas iguales & 0,723 \\
\hline \multirow[t]{2}{*}{ L. Castellana } & $\mathrm{H}$ & 59 & 7,349 & & & & \\
\hline & $\mathrm{M}$ & 41 & 7,427 & 6,294 & 0,014 & No se asumen varianzas iguales & 0,773 \\
\hline \multirow[t]{2}{*}{ L. Gallega } & $\mathrm{H}$ & 59 & 7,242 & & & & \\
\hline & $M$ & 41 & 7,463 & 5,437 & 0,022 & No se asumen varianzas iguales & 0,429 \\
\hline \multirow[t]{2}{*}{ Matemáticas } & $\mathrm{H}$ & 59 & 7,812 & & & & \\
\hline & $\mathrm{M}$ & 41 & 7,112 & 0,083 & 0,774 & Se asumen varianzas iguales & 0,029 \\
\hline \multirow[t]{2}{*}{$\mathrm{CSGH}$} & $\mathrm{H}$ & 59 & 6,98 & & & & \\
\hline & M & 41 & 6,663 & 2,234 & 0,138 & Se asumen varianzas iguales & 0,192 \\
\hline \multirow[t]{2}{*}{ Optativa } & $\mathrm{H}$ & 59 & 7,414 & & & & \\
\hline & M & 41 & 7,01 & 2,841 & 0,095 & Se asumen varianzas iguales & 0,192 \\
\hline
\end{tabular}

continúa 
doi: http://dx.doi.org/10.15359/ree.23-2.12

URL: http://www.una.ac.cr/educare

CORREO: educare@una.cr

\begin{tabular}{ccccccc}
\hline \multicolumn{7}{c}{ Según el hábitat } \\
\hline Grupo presentado & N & Media & F. & Sig. & Prueba de Levene & Sig. (bilateral) \\
\hline
\end{tabular}

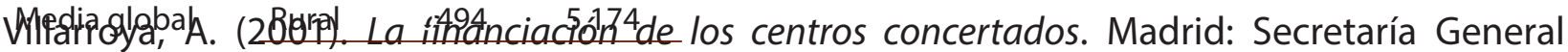

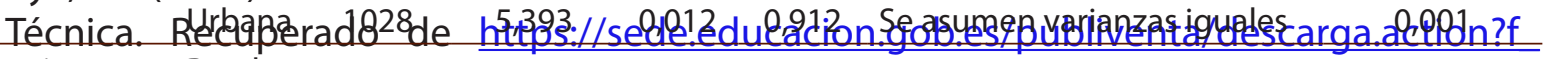

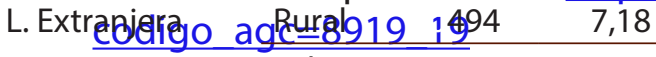

$\begin{array}{llllll}\text { Urbana } & 1028 & 7,547 & 3,251 & 0,072 & \text { Se asumen varianzas iguales } 0\end{array}$

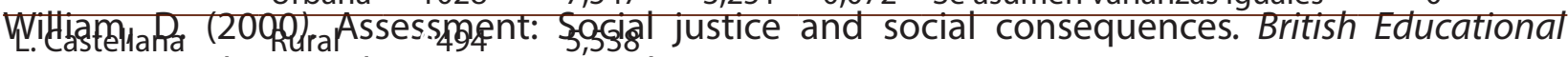

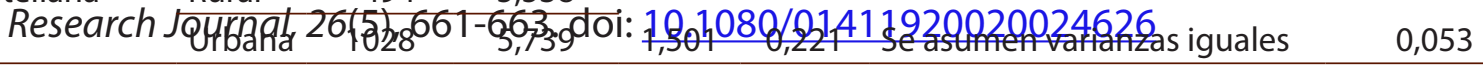

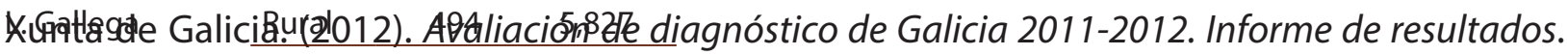

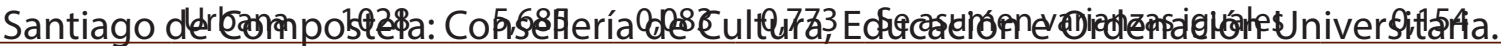

Matemáticas Rural $494 \quad 3,851$

Xunta de Galicia. Consellería de Cultura, Educación e Ordenación Universitaria. (7 de junio de

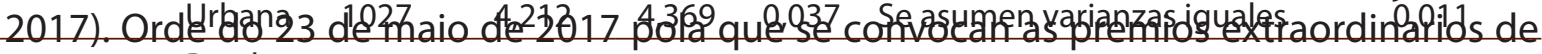

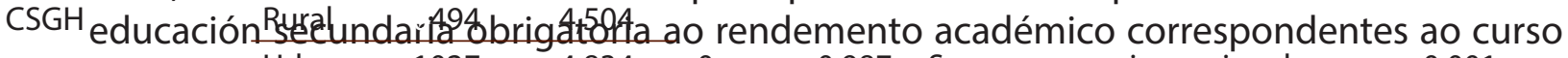

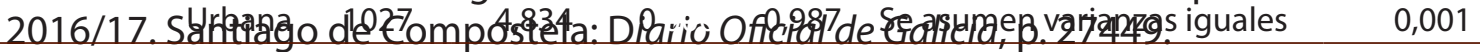

\begin{tabular}{|c|c|c|c|c|c|c|c|}
\hline \multirow[t]{2}{*}{ Optativa } & Rural & 494 & 4,092 & \multirow[b]{2}{*}{0,063} & \multirow[b]{2}{*}{0,802} & \multirow[b]{2}{*}{ Se asumen varianzas iguales } & \multirow[b]{2}{*}{0,074} \\
\hline & Urbana & 1027 & 4,319 & & & & \\
\hline Grupo ganador & & $\mathrm{N}$ & Media & F. & Sig. & Prueba de Levene & Sig. (bilateral) \\
\hline \multirow[t]{2}{*}{ Media global } & Rural & 24 & 7,596 & & & & \\
\hline & Urbana & 76 & 7,416 & 1,908 & 0,17 & Se asumen varianzas iguales & 0,182 \\
\hline \multirow[t]{2}{*}{ L. Extranjera } & Rural & 24 & 8,367 & & & & \\
\hline & Urbana & 76 & 8,288 & 0,016 & 0,898 & Se asumen varianzas iguales & 0,699 \\
\hline \multirow[t]{2}{*}{ L. Castellana } & Rural & 24 & 7,654 & & & & \\
\hline & Urbana & 76 & 7,295 & 0,483 & 0,489 & Se asumen varianzas iguales & 0,267 \\
\hline \multirow[t]{2}{*}{ L. Gallega } & Rural & 24 & 7,117 & & & & \\
\hline & Urbana & 76 & 7,401 & 1,912 & 0,17 & Se asumen varianzas iguales & 0,411 \\
\hline \multirow[t]{2}{*}{ Matemáticas } & Rural & 24 & 7,538 & & & & \\
\hline & Urbana & 76 & 7,521 & 2,574 & 0,112 & Se asumen varianzas iguales & 0,965 \\
\hline \multirow[t]{2}{*}{$\mathrm{CSGH}$} & Rural & 24 & 7 & & & & \\
\hline & Urbana & 76 & 6,803 & 0,702 & 0,404 & Se asumen varianzas iguales & 0,48 \\
\hline \multirow[t]{2}{*}{ Optativa } & Rural & 24 & 7,525 & & & & \\
\hline & Urbana & 76 & 7,161 & 0,113 & 0,738 & Se asumen varianzas iguales & 0,308 \\
\hline
\end{tabular}

continúa 


\begin{tabular}{|c|c|c|c|c|c|c|c|}
\hline \multicolumn{8}{|c|}{ Según la titularidad } \\
\hline \multicolumn{2}{|c|}{ Grupo ganador } & $\mathrm{N}$ & Media & F. & Sig. & Prueba de Levene & Sig. (bilateral) \\
\hline \multirow[t]{2}{*}{ Media global } & Privada & 582 & 5,377 & & & & \\
\hline & Pública & 940 & 5,290 & 0,899 & 0,343 & Se asumen varianzas iguales & 0,154 \\
\hline \multirow[t]{2}{*}{ L. Extranjera } & Privada & 582 & 7,669 & & & & \\
\hline & Pública & 940 & 7,279 & 4,723 & 0,03 & No se asumen varianzas iguales & 0 \\
\hline \multirow[t]{2}{*}{ L. Castellana } & Privada & 582 & 5,639 & & & & \\
\hline & Pública & 940 & 5,695 & 0,111 & 0,739 & Se asumen varianzas iguales & 0,576 \\
\hline \multirow[t]{2}{*}{ Matemáticas } & Privada & 582 & 4,134 & & & & \\
\hline & Pública & 940 & 4,066 & 0,282 & 0,595 & Se asumen varianzas iguales & 0,621 \\
\hline \multirow[t]{2}{*}{$\mathrm{CSGH}$} & Privada & 582 & 4,88 & & & & \\
\hline & Pública & 940 & 4,627 & 4,971 & 0,026 & No se asumen varianzas iguales & 0,007 \\
\hline \multirow[t]{2}{*}{ Optativa } & Privada & 410 & 3,841 & & & & \\
\hline & Pública & 765 & 3,457 & 0,853 & 0,356 & Se asumen varianzas iguales & 0,006 \\
\hline \multicolumn{2}{|c|}{ Ganadores } & $\mathrm{N}$ & Media & F. & Sig. & Prueba de Levene & Sig. (bilateral) \\
\hline \multirow[t]{2}{*}{ Media global } & Privada & 60 & 7,385 & & & & \\
\hline & Pública & 40 & 7,508 & 0,861 & 0,356 & Se asumen varianzas iguales & 0,295 \\
\hline \multirow[t]{2}{*}{ L. Extranjera } & Privada & 60 & 8,37 & & & & \\
\hline & Pública & 40 & 8,265 & 1,016 & 0,316 & Se asumen varianzas iguales & 0,553 \\
\hline \multirow[t]{2}{*}{ L. Castellana } & Privada & 60 & 7,275 & & & & \\
\hline & Pública & 40 & 7,452 & 0,005 & 0,943 & Se asumen varianzas iguales & 0,532 \\
\hline \multirow[t]{2}{*}{ L. Gallega } & Privada & 60 & 7,198 & & & & \\
\hline & Pública & 40 & 7,423 & 0,094 & 0,76 & Se asumen varianzas iguales & 0,454 \\
\hline \multirow[t]{2}{*}{ Matemáticas } & Privada & 60 & 7,27 & & & & \\
\hline & Pública & 40 & 7,695 & 0,588 & 0,445 & Se asumen varianzas iguales & 0,191 \\
\hline \multirow[t]{2}{*}{$\mathrm{CSGH}$} & Privada & 60 & 7,008 & & & & \\
\hline & Pública & 40 & 6,745 & 0,524 & 0,471 & Se asumen varianzas iguales & 0,281 \\
\hline \multirow[t]{2}{*}{ Optativa } & Privada & 60 & 7,178 & & & & \\
\hline & Pública & 40 & 7,295 & 0 & 0,997 & Se asumen varianzas iguales & 0,707 \\
\hline
\end{tabular}

continúa 
doi: http://dx.doi.org/10.15359/ree.23-2.12

URL: http://www.una.ac.cr/educare

CORREO: educare@una.cr

\begin{tabular}{|c|c|c|c|c|c|c|c|}
\hline \multicolumn{8}{|c|}{ Según los estudios universitarios de los padres } \\
\hline \multicolumn{2}{|c|}{ Grupo presentado } & $\mathrm{N}$ & Media & F. & Sig. & Prueba de Levene & Sig. (bilateral) \\
\hline \multirow[t]{2}{*}{ Media global } & $\mathrm{Si}$ & 1075 & 5,413 & & & & \\
\hline & No & 447 & 5,103 & 0,35 & 0,554 & Se asumen varianzas iguales & 0 \\
\hline \multirow[t]{2}{*}{ L. Extranjera } & $\mathrm{Si}$ & 1075 & 7,547 & & & & \\
\hline & No & 447 & 7,141 & 2,44 & 0,119 & Se asumen varianzas iguales & 0 \\
\hline \multirow[t]{2}{*}{ L. Castellana } & $\mathrm{Si}$ & 1075 & 5,761 & & & & \\
\hline & No & 447 & 5,465 & 0,912 & 0,34 & Se asumen varianzas iguales & 0,006 \\
\hline \multirow[t]{2}{*}{ L. Gallega } & $\mathrm{Si}$ & 1075 & 5,793 & & & & \\
\hline & No & 447 & 5,583 & 0,201 & 0,654 & Se asumen varianzas iguales & 0,04 \\
\hline \multirow[t]{2}{*}{ Matemáticas } & $\mathrm{Si}$ & 1074 & 4,243 & & & & \\
\hline & No & 447 & 3,738 & 3,955 & 0,047 & No se asumen varianzas iguales & 0 \\
\hline \multirow[t]{2}{*}{$\mathrm{CSGH}$} & $\mathrm{Si}$ & 1074 & 4,827 & & & & \\
\hline & No & 447 & 4,486 & 0,019 & 0,89 & Se asumen varianzas iguales & 0,001 \\
\hline \multirow[t]{2}{*}{ Optativa } & $\mathrm{Si}$ & 1074 & 4,27 & & & & \\
\hline & No & 447 & 4,186 & 0,056 & 0,814 & Se asumen varianzas iguales & 0,518 \\
\hline \multicolumn{2}{|l|}{ Grupo ganador } & $\mathrm{N}$ & Media & F. & Sig. & Prueba de Levene & Sig. (bilateral) \\
\hline \multirow[t]{2}{*}{ Media global } & $\mathrm{Si}$ & 84 & 7,473 & & & & \\
\hline & No & 16 & 7,388 & 0,054 & 0,816 & Se asumen varianzas iguales & 0,589 \\
\hline \multirow[t]{2}{*}{ L. Extranjera } & $\mathrm{Si}$ & 84 & 8,396 & & & & \\
\hline & No & 16 & 7,838 & 0,522 & 0,472 & Se asumen varianzas iguales & 0,017 \\
\hline \multirow[t]{2}{*}{ L. Castellana } & $\mathrm{Si}$ & 84 & 7,385 & & & & \\
\hline & No & 16 & 7,363 & 1,146 & 0,287 & Se asumen varianzas iguales & 0,954 \\
\hline \multirow[t]{2}{*}{ L. Gallega } & $\mathrm{Si}$ & 84 & 7,339 & & & & \\
\hline & No & 16 & 7,3 & 1,884 & 0,173 & Se asumen varianzas iguales & 0,923 \\
\hline \multirow[t]{2}{*}{ Matemáticas } & $\mathrm{Si}$ & 84 & 7,527 & & & & \\
\hline & No & 16 & 7,513 & 0,296 & 0,588 & Se asumen varianzas iguales & 0,973 \\
\hline \multirow[t]{2}{*}{$\mathrm{CSGH}$} & $\mathrm{Si}$ & 84 & 6,849 & & & & \\
\hline & No & 16 & 6,856 & 2,031 & 0,157 & Se asumen varianzas iguales & 0,982 \\
\hline \multirow[t]{2}{*}{ Optativa } & $\mathrm{Si}$ & 84 & 7,22 & & & & \\
\hline & No & 16 & 7,394 & 0,506 & 0,479 & Se asumen varianzas iguales & 0,677 \\
\hline
\end{tabular}

Nota: Elaboración propia a partir de datos facilitados por la Consellería de Cultura (Xunta de Galicia, 2012.) 